\title{
Quantitative evaluation of fetal ventricular function by speckle tracking echocardiography
}

\author{
Ling Luo ${ }^{1}$, Hanmin $\mathrm{Liu}^{2}$, Shu Zhou ${ }^{2}$, Fuming Zhao ${ }^{2}$, Qi Zhu ${ }^{3}$, nan guo ${ }^{2}$, and Jiao Chen ${ }^{2}$ \\ ${ }^{1}$ Sichuan University West China Second University Hospital \\ ${ }^{2}$ Affiliation not available \\ ${ }^{3}$ West China Second University Hospital,West China Medcical School,Sichuan Univeisity
}

April 18, 2021

\begin{abstract}
Methods The study included 59 patients with normal fetal heart structure, blood flow, and heart rhythm (fetal abnormalitynegative group) and 50 patients with abnormal fetal heart structure, blood flow, and/or heart rhythm (fetal abnormalitypositive group). aCMQ was performed in both groups to obtain left and right ventricular endocardial global longitudinal strain (GLSendo), mid-myocardial global longitudinal strain (GLSmid), and epicardial global longitudinal strain (GLSepi). Parameters between the two groups were compared and correlation analyses performed. A deformation analysis was performed by two trained observers, and reproducibility was assessed. Results The fetal left ventricular and right ventricular global longitudinal strain (LV-GLS and RV-GLS, respectively) decreased in a gradient from the endocardium to the epicardium. LV-GLS and RV-GLS of all myocardial layers were lower in the fetal abnormality-positive than -negative group (all $\mathrm{P}<0.05$ ). Correlation analysis showed that neither LV-GLS nor RV-GLS was significantly correlated with gestational age in the fetal abnormality-negative group (all P>0.05), whereas left ventricular GLSendo, GLSmid, and GLSepi were negatively correlated with gestational age in the fetal abnormality-positive group $(\mathrm{r}=-0.39$ to -0.44 , all $\mathrm{P}<0.05)$. Repeatability testing showed that the inter-observer and intra-observer intraclass correlation coefficients for LV-GLS and RV-GLS in each myocardial layer were $>0.75$ (all $\mathrm{P}<0.001$ ). Conclusions As a new speckle tracking echocardiography tool, aCMQ has feasibility and repeatability in evaluating myocardial deformation of the fetal ventricle. This technique might provide helpful information on ventricular myocardial deformation in fetal hearts with abnormal structure or rhythm for clinical guidance in pregnancy.
\end{abstract}

\section{Hosted file}

Evaluation of fetal ventricular function by STE.pdf available at https://authorea.com/users/ 408571/articles/518521-quantitative-evaluation-of-fetal-ventricular-function-by-speckletracking-echocardiography 

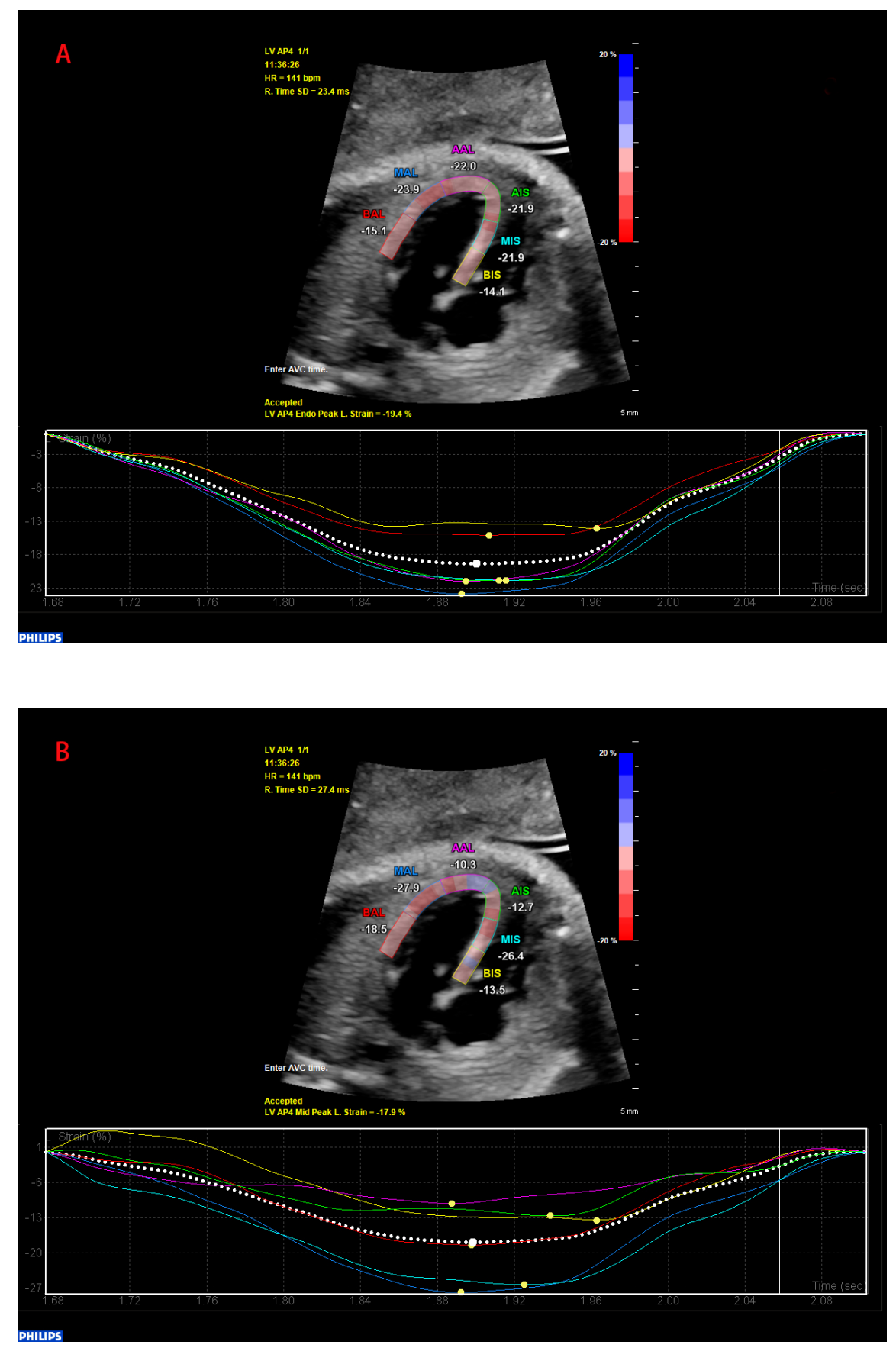



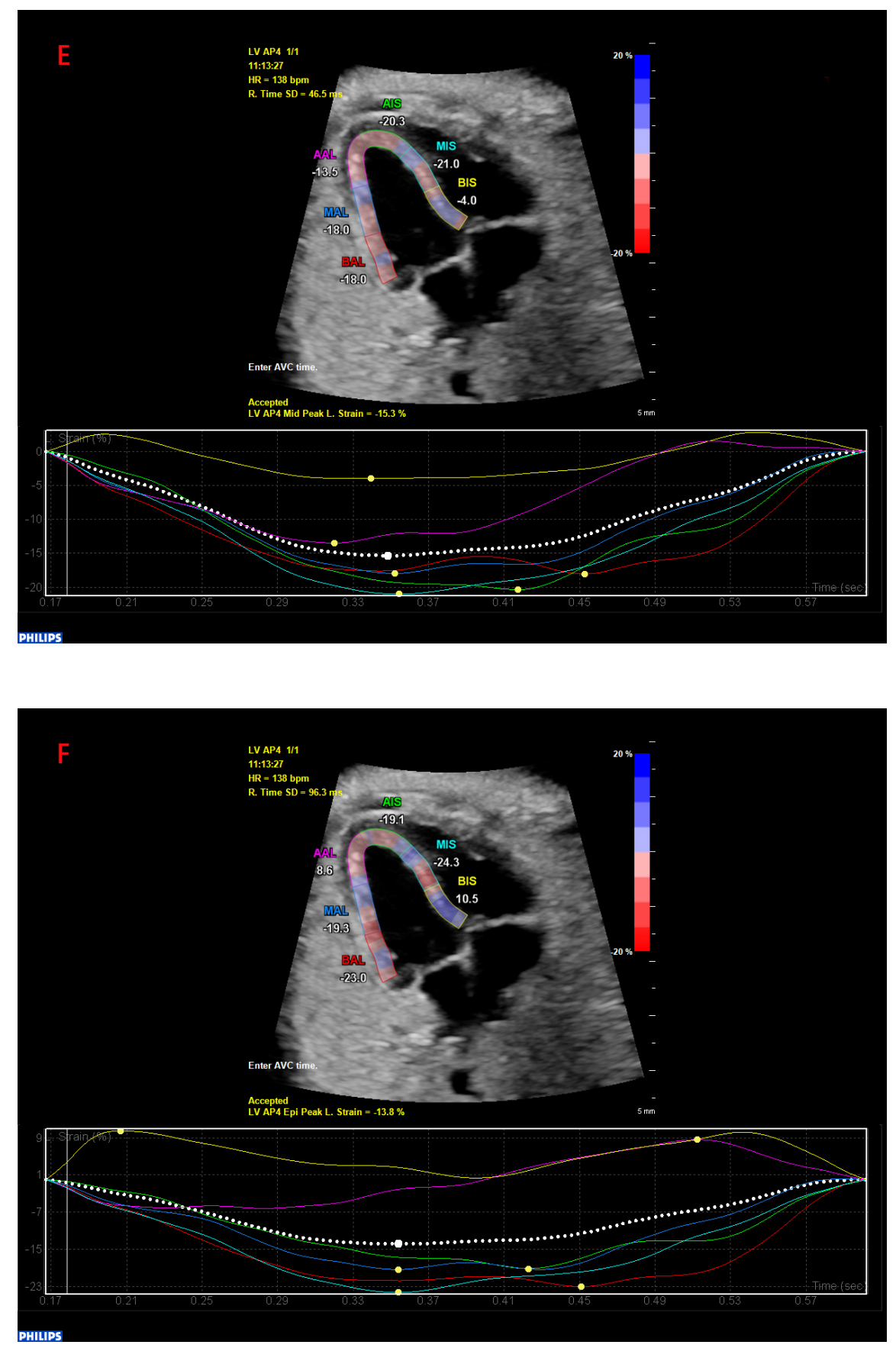

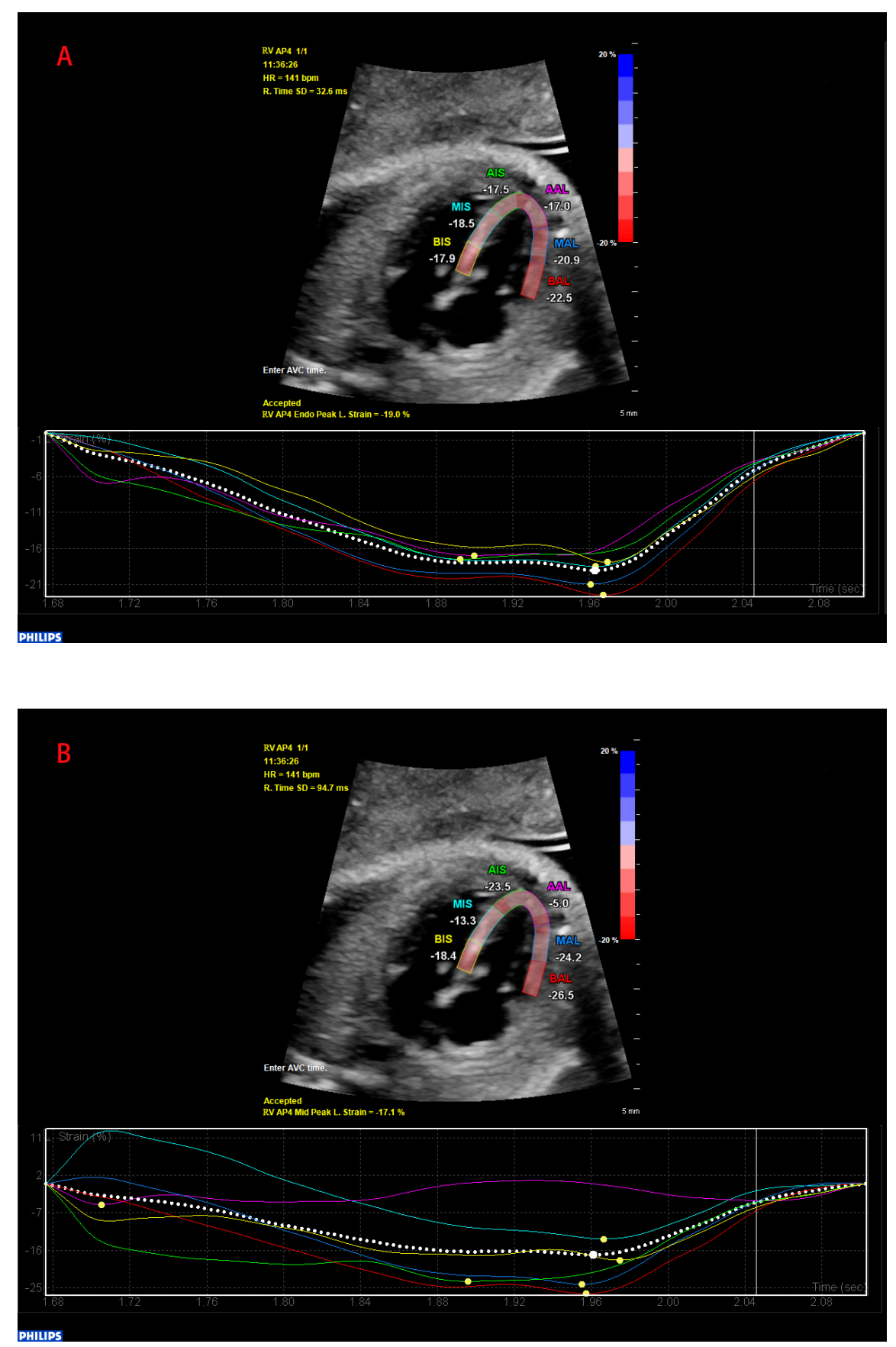

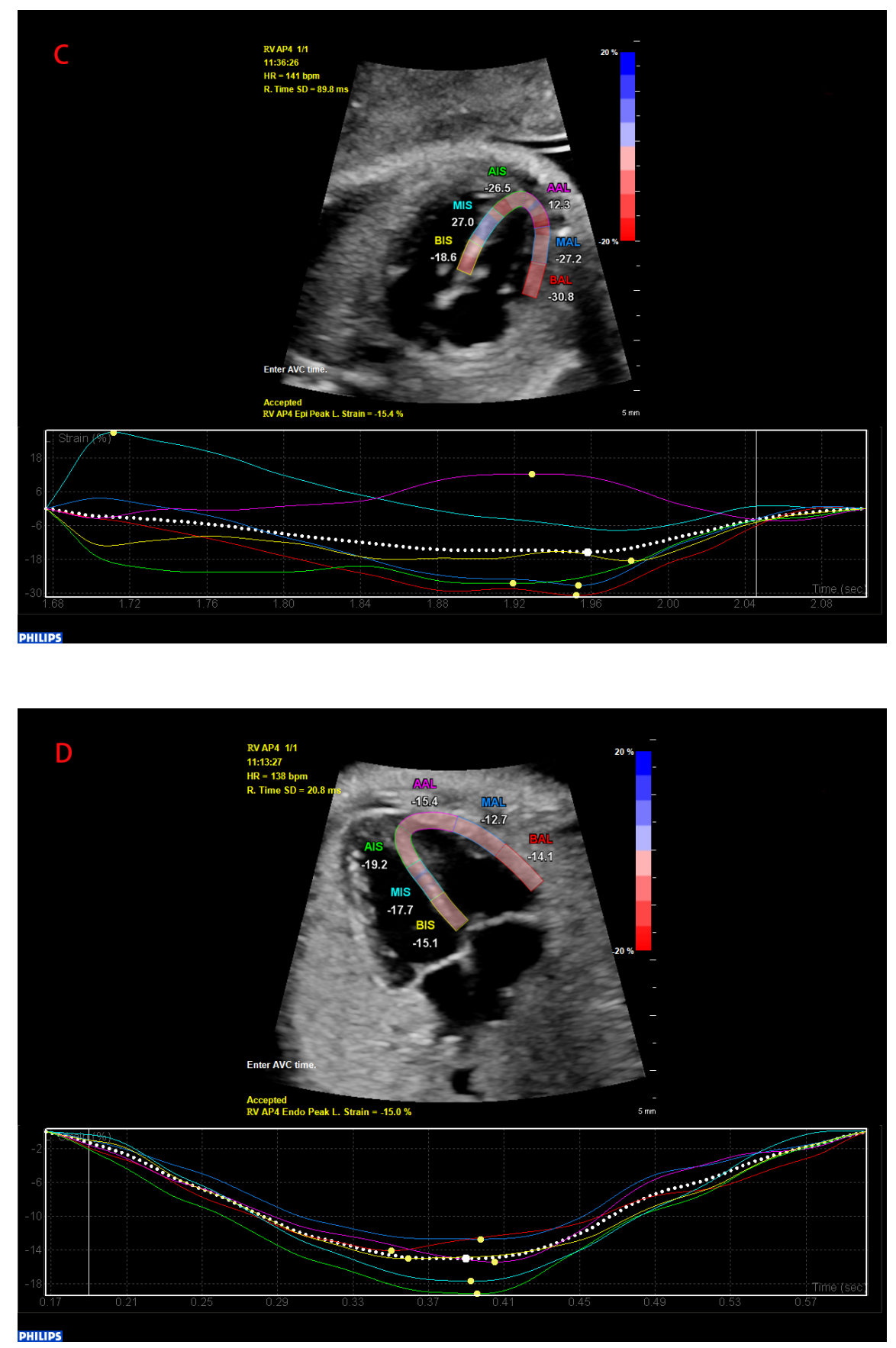

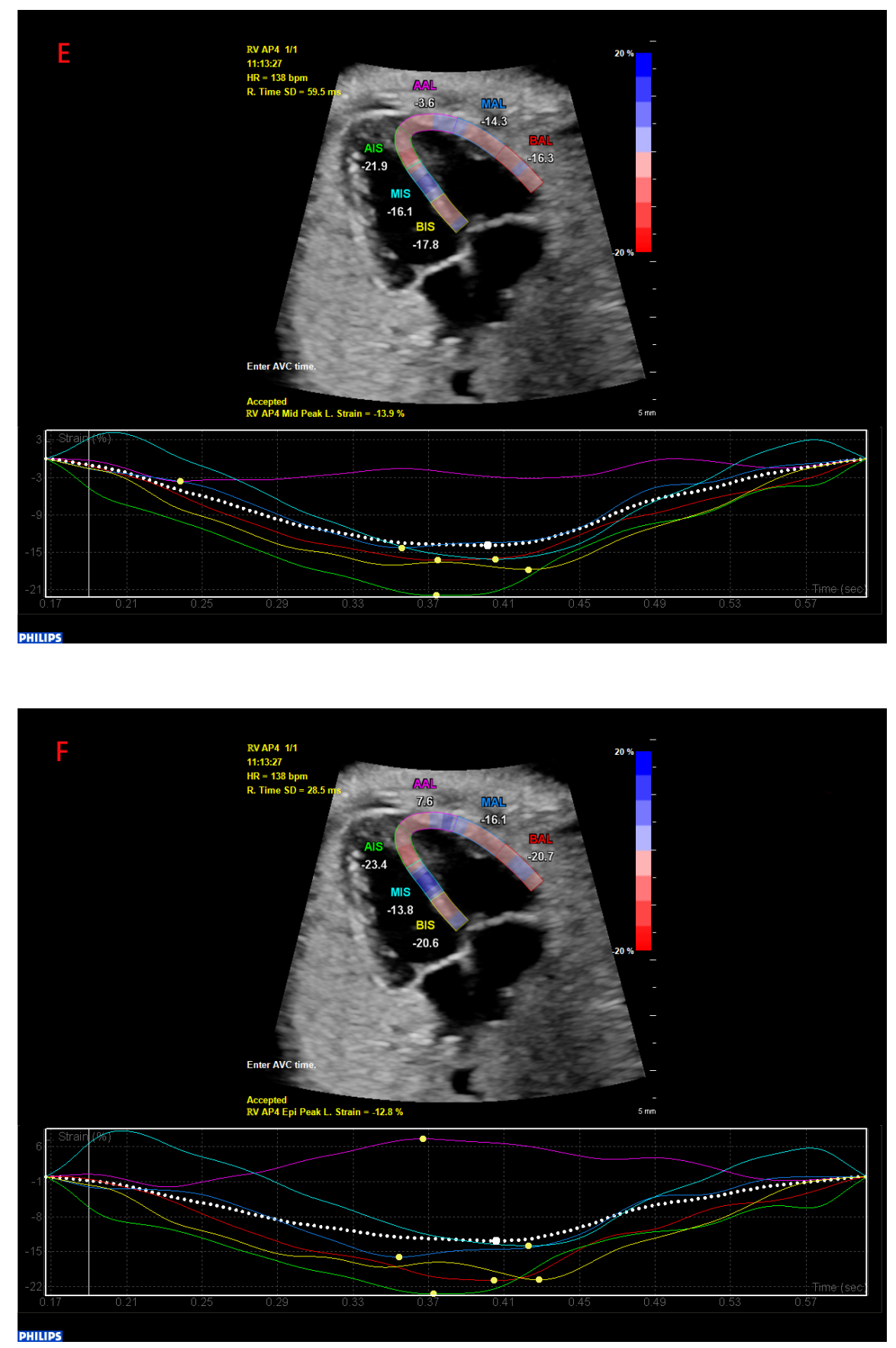

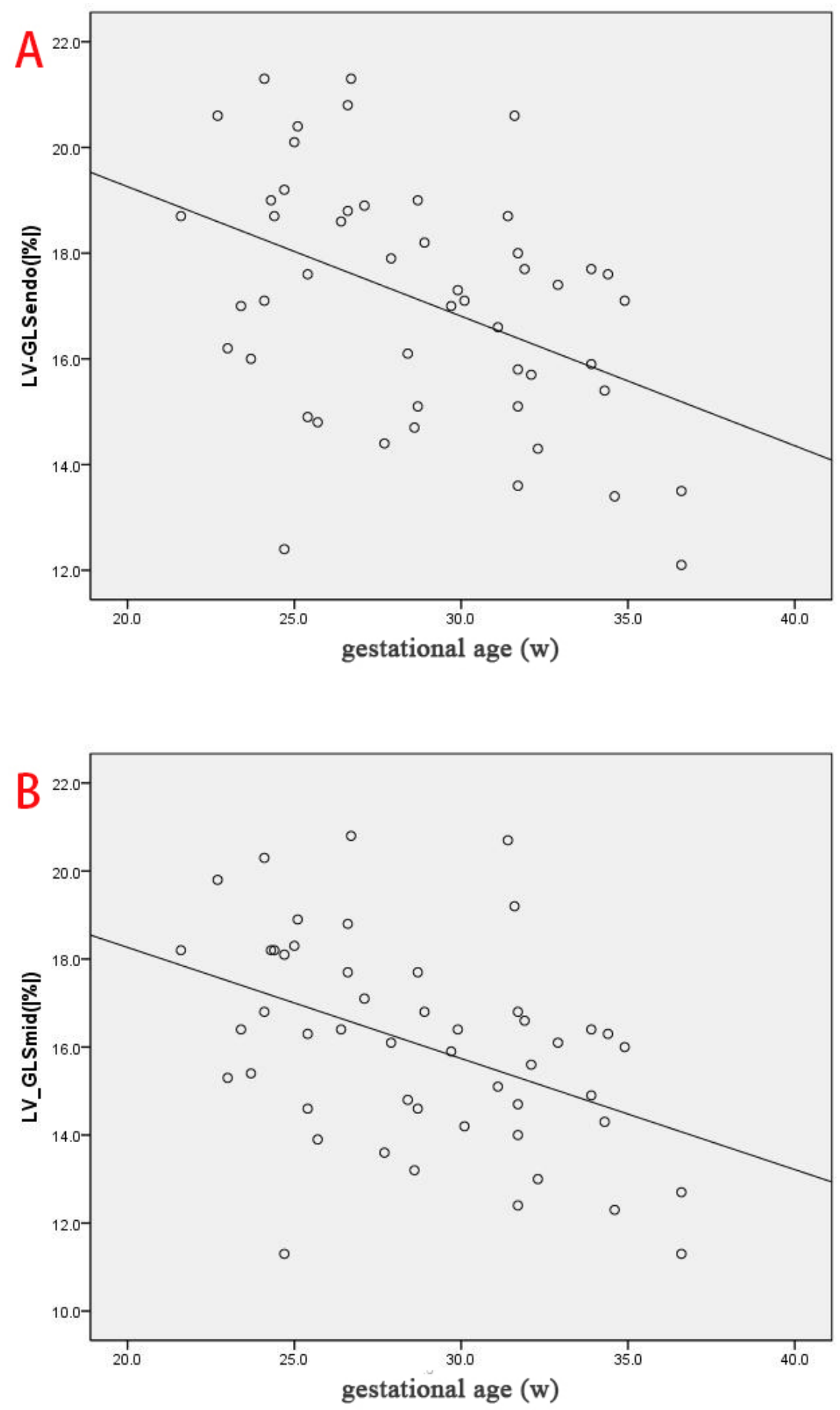


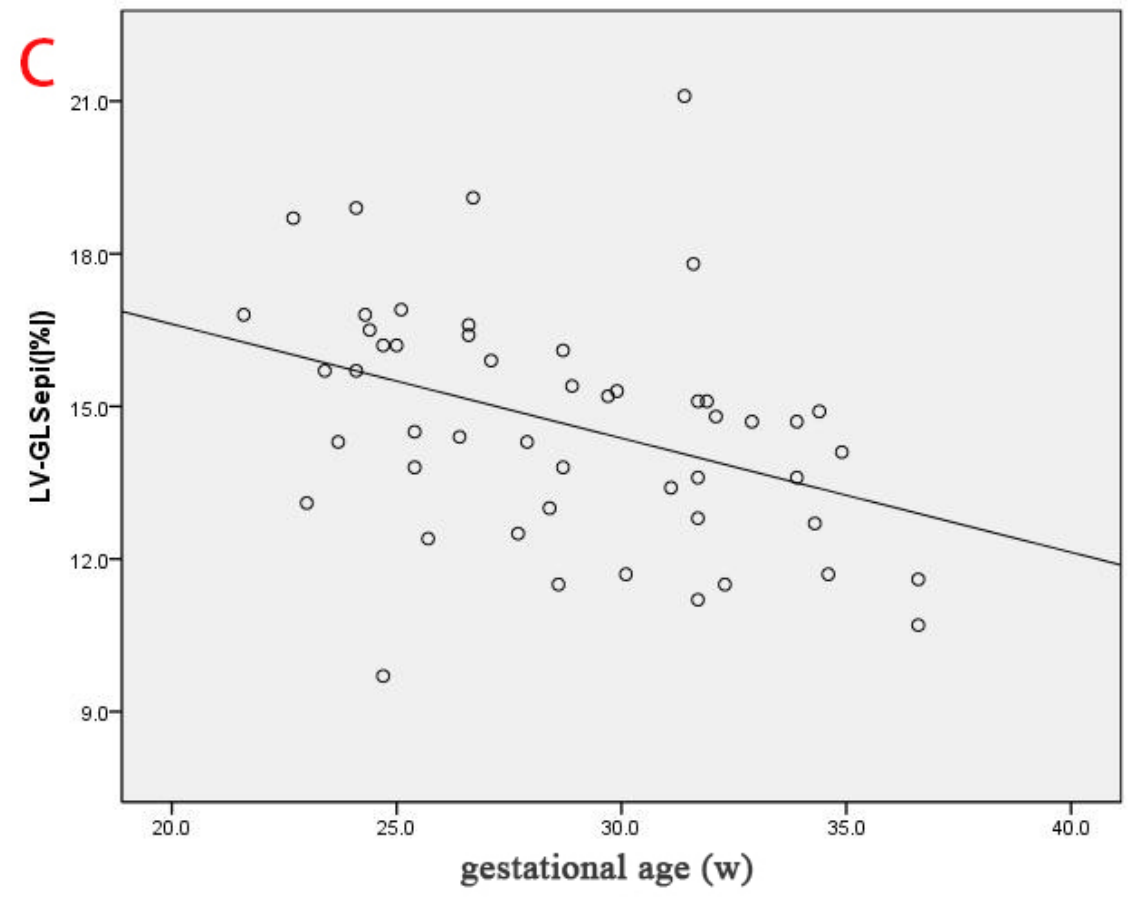

